\title{
Combination of the FGFR4 inhibitor PD173074 and 5-fluorouracil reduces proliferation and promotes apoptosis in gastric cancer
}

\author{
YAN-WEI YE ${ }^{1,2 *}$, SHUANG HU ${ }^{2,3^{*}}$, YING-QIANG SHI ${ }^{4,5}$, XIE-FU ZHANG ${ }^{1,2}$, YE ZHOU $^{4,5}$, \\ CHUN-LIN ZHAO ${ }^{1,2}$, GUO-JUN WANG ${ }^{1,2}$, JIAN-GUO WEN ${ }^{2}$ and HONG ZONG ${ }^{2,3}$ \\ ${ }^{1}$ Department of Gastrointestinal Surgery, ${ }^{2}$ Institute of Clinical Medicine, and ${ }^{3}$ Department of Oncology, \\ The First Affiliated Hospital of Zhengzhou University, Zhengzhou, Henan; ${ }^{4}$ Department of Stomach \\ and Soft Tissue Sarcoma Surgery, Shanghai Cancer Center, Fu Dan University, Shanghai; \\ ${ }^{5}$ Department of Oncology, Shanghai Medical College, Fu Dan University, Shanghai, P.R. China
}

Received August 19, 2013; Accepted September 20, 2013

DOI: $10.3892 /$ or.2013.2796

\begin{abstract}
Our previous findings revealed that FGFR4 may be a novel therapeutic target for gastric cancer. The aim of the present study was to explore the effects of a combination of PD173074 (PD) and 5-fluorouracil (5-Fu) on the biological behavior of gastric cancer cell lines and the relevant mechanisms involved. MKN45, a gastric cancer cell line, was treated with each single agent alone or a combination of FGF19, PD and 5-Fu. Then, a series of functional assays were performed using CCK-8 assay and flow cytometry. Western blot analysis was used to determine the expression of signaling pathway and downstream-related molecules in the MKN45 cells following the different treatments. As the concentration of PD and $5-\mathrm{Fu}$ increased, the cell viability gradually decreased; the viability of the combination group was less than the viability following single administration. Western blot analysis showed that FGFR4 expression was weak in the 5-Fu-treated groups when compared with the control. PD markedly increased the apoptosis rate of MKN45 cells when compared to the control; the apoptosis rate in the cells treated with the combination of $\mathrm{PD}$ and 5-Fu was higher than that in the cells following single
\end{abstract}

Correspondence to: Dr Yan-Wei Ye, Department of Gastrointestinal Surgery, The First Affiliated Hospital of Zhengzhou University, 1 Jian-She Road, Zhengzhou, Henan 450052, P.R. China E-mail: yeyanwei@hotmail.com

Dr Hong Zong, Department of Oncology, The First Affiliated Hospital of Zhengzhou University, 1 Jian-She Road, Zhengzhou, Henan 450052, P.R. China

E-mail: zonh@126.com

*Contributed equally

Abbreviations: FGFR4, fibroblast growth factor receptor 4; PD, PD173074; 5-Fu, 5-fluorouracil; CCK-8, Cell Counting Kit-8; GC, gastric cancer

Key words: gastric cancer, 5-fluorouracil, FGFR4, MKN45, PD173074 treatment. Furthermore, PD reduced the expression of p-ERK and $\mathrm{Bcl}-\mathrm{xl}$ and increased caspase- 3 expression. Inhibition of the activity of FGFR4 may be the main mechanisms of PD effect while 5-Fu reduced FGFR4 expression. Furthermore, the effects of the combination of 5-Fu and PD in inhibiting proliferation, increasing apoptosis and arresting cell cycle were superior to these effects following the single agent treatments, suggesting that the two drugs applied in combination may contribute to the effective treatment of gastric cancer.

\section{Introduction}

Gastric cancer (GC) is the fourth most common malignant tumor worldwide (1). GC patients in China have an extremely high rate of morbidity and mortality. Patients with earlystage GC account for only $20 \%$ of cases in China, which is much less than that in Japan and Korea. Therefore, most GC patients in China are diagnosed with advanced $\mathrm{GC}$ and require comprehensive therapy including surgery, radiotherapy and chemotherapy. Currently, there are many neoadjuvant, adjuvant and palliative chemotherapies available for GC patients, such as ECF, DCF, oxaliplatin and 5-fluorouracil (5-Fu). However, the most crucial drug is 5-Fu. Recently, targeted drugs have become an intense field in the research and application of GC treatment. Cetuximab (targeted to EGFR), bevacizumab (targeted to VEGF) and Herceptin (targeted to Her-2) have been applied in clinical trails of gastric cancer. Importantly, in October 2010, the Food and Drug Administration approved the combination of Herceptin and chemotherapy for the treatment of late-stage or metastatic GC patients with positive expression of Her-2.

However, to date, no other targeted drugs have achieved a breakthrough in the treatment of GC apart for Herceptin. Although targeted drug treatment is the future direction, exploring new therapeutic targets in GC has become an essential goal. Katoh (2) suggested that the FGFR family may be important in clinical cancer diagnostics and therapeutics. Several literature reports indicate that FGFR4, a member of the FGFR family, has a crucial role in tissue repair and embryonic development $(3,4)$. However, the role of FGFR4 in GC has not been fully clarified. 
Our previous research showed that the mRNA expression of FGFR4 was markedly increased in gastric cancer tissue when compared with that in corresponding normal tissue as detected by real-time PCR. The FGFR4 Arg388 genotype, a marker for GC progression, was suggested to predict prognosis in GC (5). Furthermore, a series of functional assays in vitro, utilizing small interfering RNA, were carried out, including proliferation assay, clone assay and apoptosis detection. The results demonstrated that knockdown of FGFR4 expression led to a decrease in the proliferative ability and an increase in the apoptosis rate in the MKN45 and SGC7901 GC cell lines. Moreover, western blot analysis demonstrated that the expression of caspase-3 was observably increased while Bcl-xl expression was markedly decreased in MKN45 and SGC7901 cells following FGFR4-siRNA transfection. Therefore, FGFR4 may contribute to GC progression through regulation of proliferation and anti-apoptosis, indicating that FGFR4 may be used as a novel drug target against GC (6).

In order to further clarify the clinical value of FGFR4 expression in GC and explore new targeted drugs, PD173074 (PD), a FGFR inhibitor, was introduced in the present study. In terms of the application of Herceptin, we first investigated whether a single agent treatment and the combination of $5-\mathrm{Fu}$ and PD influence the biological behavior of GC cells using a series of functional research methods in vitro, including proliferation assay, apoptosis detection, assessment of cell cycle distribution as well as determination of the expression of cell signal pathway and downstream effector molecules by western blot analysis. Furthermore, to verify whether PD has an impact on GC cells by inhibiting FGFR4, FGF19, a special agonist of FGFR4, was applied in the present study. Through a series of functional assays, we aimed to clarify the mechanism of PD and 5-Fu effects on GC cells. We suggest that PD has the potential to become a new targeted drug similar to Herceptin and could be applied for the treatment of GC patients.

\section{Materials and methods}

Antibodies and reagents. The rabbit polyclonal anti-FGFR4 antibody was obtained from Santa Cruz Biotechnology (Santa Cruz, CA, USA). Rabbit monoclonal anti-Bcl-xl, antiAkt, anti-phospho-Akt, anti-phospho-ERK, anti-caspase-3 and anti-GAPDH antibodies were all purchased from Cell Signaling Technology (Beverly, MA, USA). Secondary horseradish peroxidase-conjugated antibodies included goat anti-mouse and goat anti-rabbit from Sigma-Aldrich Corp. (St. Louis, MO, USA). PD173074 (P2499) was purchased from Sigma-Aldrich Corp. (Shanghai, China), and recombinant human FGF19 was obtained from PeproTech Inc. (Rocky Hill, NJ, USA). 5-Fu was provided by the clinical trial group in our research center.

Cell lines and cell culture. Human gastric cancer cell lines, SNU-1 and SNU-16, were purchased from the American Type Culture Collection (Manassas, VA, USA). MKN45 and SGC7901 cell lines were obtained from the Chinese Academy of Sciences, the Sciences Cell Bank of the Type Culture Collection (CBTCCCAS, Shanghai, China). Cell lines were cultivated in RPMI-1640 medium (Gibco, Grand Island, NY, USA) supplemented with $10 \%$ fetal bovine serum (FBS;
Gibco), $100 \mathrm{U} \mathrm{ml}^{-1}$ of penicillin and $100 \mu \mathrm{g} \mathrm{ml}^{-1}$ of streptomycin (Caisson Laboratories, Inc., North Logan, UT, USA) at $37^{\circ} \mathrm{C}$ in a humidified atmosphere containing $5 \% \mathrm{CO}_{2}$.

Reverse transcriptase-PCR. According to the protocol supplied by Invitrogen (San Diego, CA, USA), TRIzol was used to extract total RNA from the GC cell lines. RevertAid ${ }^{\mathrm{TM}}$ First Strand cDNA synthesis kit (MBI, Fermantas, Burlington, ON, Canada) was used to reverse transcribe $1 \mu \mathrm{g}$ of each RNA sample into cDNA in a total volume of $20 \mu \mathrm{l}$. Primers consisted of: FGFR-4, 5'-AGATGCTCAAAGACAACGCCT-3' and 5'-CGCACTCCACGATCACGTA-3'; GAPDH 5'-GAAGAT GGTGATGGGATTTC-3' and 5'-GAAGGTGAAGGTCGG AGTC-3'. Taq 2X PCR Master Mix (Tiangen Biotech, Co., Ltd., Beijing, China) was used for PCR amplication. The annealing temperature of FGFR 4 was $57^{\circ} \mathrm{C}$. PCR products were subjected to electrophoresis on a $2 \%$ agarose gel and were visualized by ethidium bromide staining.

Quantitative real-time PCR. Gene specific primers for FGFR4 and GAPDH were the same as those used for PCR in the present study. Specificity of real-time PCR primers was checked by melting curve analysis and by loading PCR products on agarose gel. Four gastric cancer cell line specimens were used to carry out real-time PCR in a final reaction volume of $20 \mu \mathrm{l}$ according to the protocol supplied by Takara (Shiga, Japan). The experiment was carried out in duplicate. GAPDH was used as a housekeeping control for possible differences in cDNA amounts. Relative differences (-fold) were calculated according to the comparative $\mathrm{Ct}$ method.

Protein extraction and western blotting. Cells were harvested following treatment for $72 \mathrm{~h}$, and whole-cell lysates were prepared using the Mammalian Protein Extraction reagent (Merck, Darmstadt, Germany) in accordance with the manufacturer's instructions. Protein concentrations of the samples were determined by the bicinchoninic acid (BCA) protein assay (Pierce, Rockford, IL, USA). Protein samples (40 $\mu \mathrm{g}$ of each protein) boiled for $5 \mathrm{~min}$ were separated on 10\% SDS-polyacrylamide gels and transferred onto PVDF membranes. The membranes were blocked for $1 \mathrm{~h}$ at room temperature with phosphate-buffered saline (PBS) containing $0.05 \%$ Tween-20 and 5\% non-fat dried milk, and incubated overnight at $4^{\circ} \mathrm{C}$ with the primary antibodies following the manufacturer's recommended conditions. Immunoblots were washed three times with PBS containing 0.05\% Tween-20 and $1 \%$ non-fat milk and incubated with secondary antibodies conjugated with horseradish peroxidase against mouse $\mathrm{IgG}$ or rabbit $\mathrm{IgG}$ for $1 \mathrm{~h}$ at room temperature. Immunoreactive proteins were visualized using the ECL detection system (ImageQuant LAS 3000; General Electric Co., Fairfield, CT, USA). Three independent western blot assays were performed for all samples.

Proliferation assay. The effect of each compound on the proliferation of GC cells was determined by Cell Counting Kit-8 (CCK-8) (Dojindo Laboratories, Kumamoto, Japan) according to the instructions provided by the manufacturer. Cell viability following treatment with 5-Fu, PD and FGF19 was also determined using the CCK-8. The concentrations of 


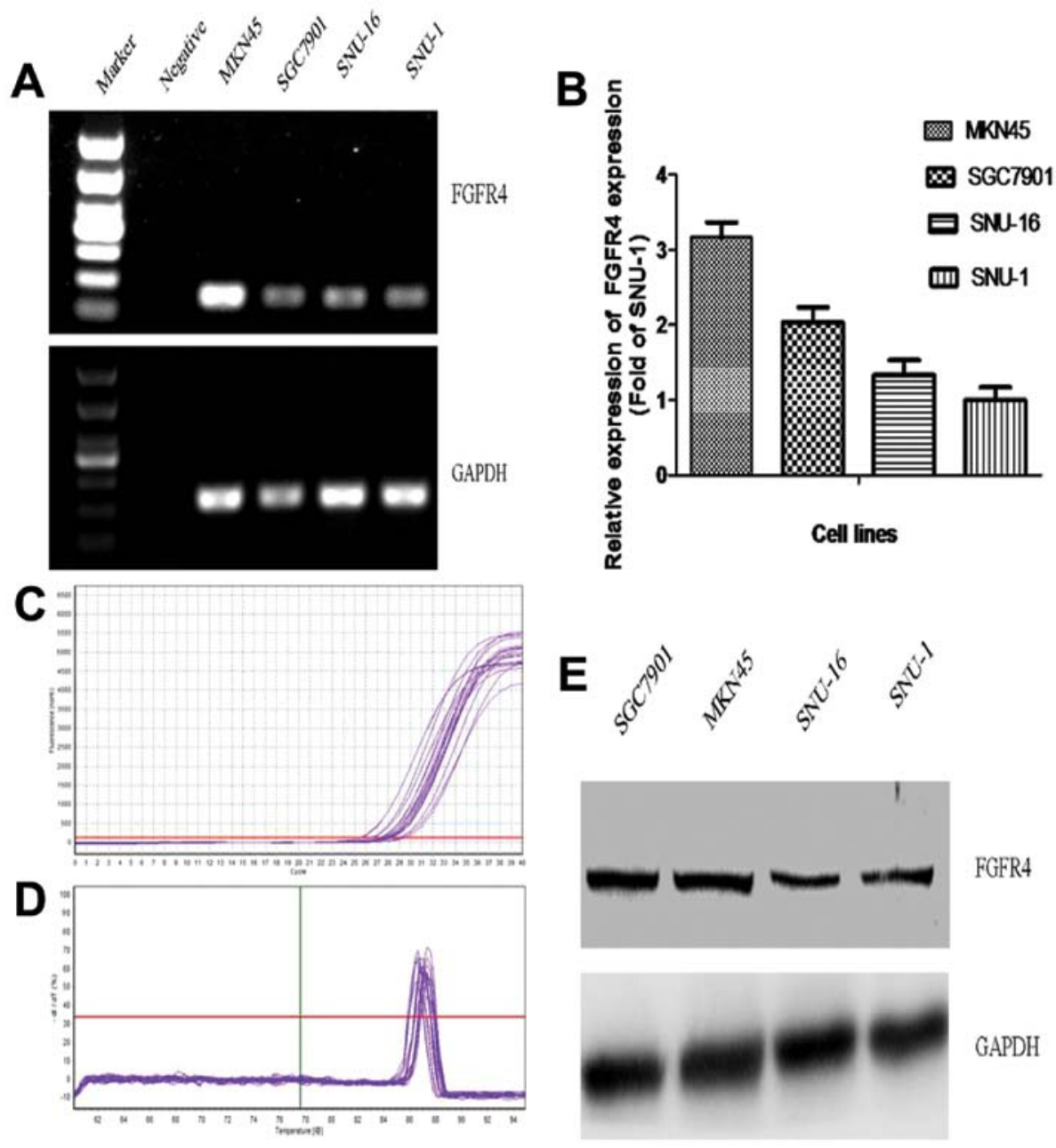

Figure 1. Expression of FGFR4 mRNA and protein in gastric cancer cell lines, MKN45, SGC7901, SNU-1 and SNU-16. Expression of FGFR4 mRNA in the different GC cell lines was revealed by (A) RT-PCR and (B) quantitative real-time PCR. (C and D) Illustrated melting curve and quantification curve of FGFR4, respectively. (E) Expression of FGFR4 protein in the different GC cell lines was assessed by western blot analysis; GAPDH served as the loading control. Expression of FGFR4 mRNA and protein in MKN45 cells was the highest among the 4 GC cell lines.

each compound were as follows in order to choose a suitable concentration for a series of assays; PD173074 (0, 1.25, 2.5, 5, 10 and $20 \mu \mathrm{M})$ and FGF19 $(0,12.5,25,50,100$ and $200 \mathrm{ng} / \mathrm{ml})$ were studied; 5-Fu $(0,50,100,200,400$ and $800 \mu \mathrm{M})$ was used based on a previous study (7). Cells, treated with the different compounds at the indicated concentrations for $48 \mathrm{~h}$, were detached using trypsin, and the number of cells was counted using a hematocytometer counting chamber (VWR International, Darmstadt, Germany). Cells (2x10\%3ell), were then incubated in 96-well culture plates (Corning Inc., New York, NY, USA) in $100 \mu \mathrm{l}$ of medium. After culturing for 1, 2, 3, 4 and 5 days, the supernatant was removed, and cell growth was detected using CCK-8 according to the manufacturer's instructions. Absorbance was measured at $450 \mathrm{~nm}$ using a microplate reader. The percentage of cell viability was determined as the ratio of the absorbance of the sample vs. the control. The $\mathrm{IC}_{50}$ of the reagents was determined as the concentration of each reagent exhibiting $50 \%$ cell growth inhibition as compared with the control cell growth. Six replicate wells were used for each reagent concentration. All experiments were performed in triplicate and repeated at least three times.
Assay of apoptosis. Cells were treated with each agent alone or combinations of PD, 5-Fu and FGF19 at the suitable concentration for $24 \mathrm{~h}$. Then the cells were harvested. Annexin $\mathrm{V}$ and propidium iodide (PI) for flow cytometry were purchased from Invitrogen (catalog no. V13241, USA) for detecting apoptosis. A working solution of $5 \mu \mathrm{l}$ of Annexin $\mathrm{V}$ and $1 \mu \mathrm{l} 100 \mu \mathrm{g} / \mathrm{ml}$ PI was added to each $100 \mu \mathrm{l}$ of the cell suspension. The cells were incubated at room temperature for $15 \mathrm{~min}$. Subsequently, $400 \mu 1$ 1X Annexin-binding buffer was added, gently mixed and the sample was kept on ice in accordance with the manufacturer's instructions. Thereafter, all samples were analyzed by a FACSCalibur flow cytometer with CellQuest software (BD Biosciences, Mountain View, CA, USA).

Statistical analysis. Statistical analysis was performed with SPSS 13.0 software (SPSS Inc., Chicago, IL, USA). Data are expressed as means $\pm \mathrm{SD}$, and three individual experiments were carried out in triplicate. The Student's t-test was used to compare data between two groups. One-way ANOVA and Dunnett's test were used to compare data between three or more groups. $\mathrm{P}<0.05$ was considered to indicate a statistically significant result. 

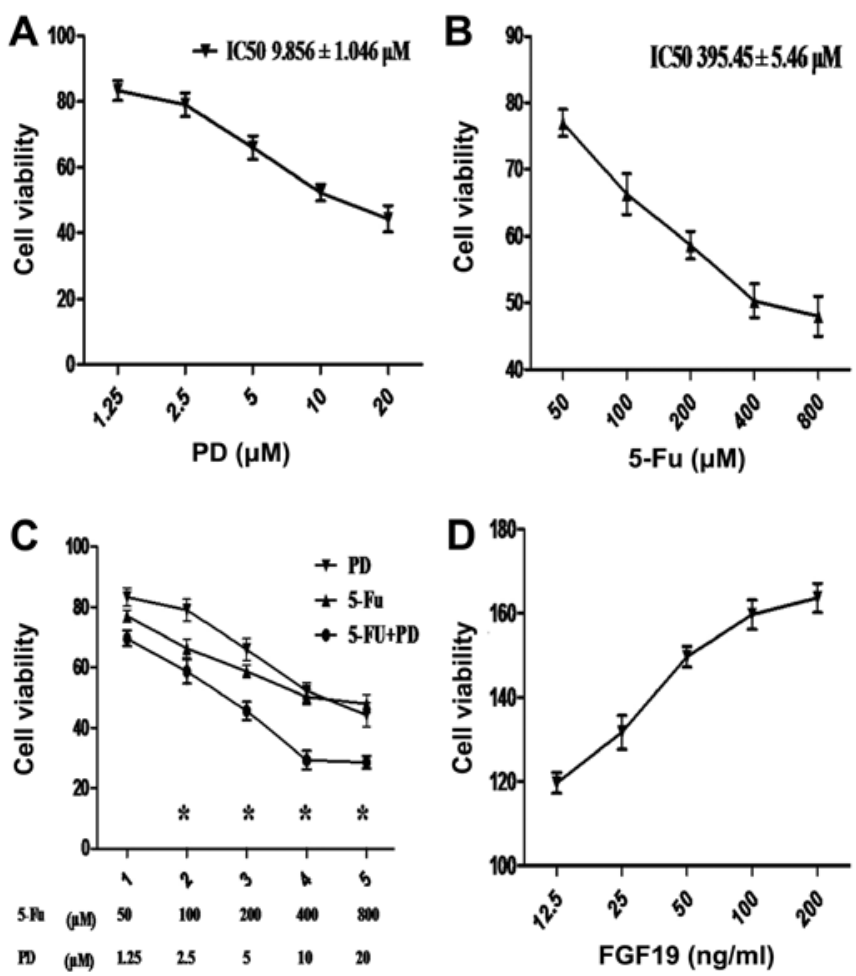

Figure 2. Effects on the cell viability of MKN45 cells of the different reagents using CCK- 8 . The cell viability of the MKN45 cells progressively declined with increasing concentrations of (A) PD and (B) 5-Fu. (C) The inhibitory effect on the cell viability of MKN45 cells was more significant following treatment with the combination of 5-Fu and PD than that following single agent treatment with the two agents (one-way ANOVA and Dunnett's test; $\left.{ }^{*} \mathrm{P}<0.05\right)$. (D) As the concentration of FGF19 increased, the cell viability gradually rose.

\section{Results}

FGFR4 is expressed in the gastric cancer cell lines. Expression of FGFR4 in the gastric cancer cell lines, at the mRNA and protein levels, was evaluated using reverse transcription PCR, quantitative real-time PCR and western blot analysis. As shown in Fig. 1A, expression of FGFR4 mRNA in the MKN45 cells was much stronger than these levels in the other 3 common GC cell lines, SGC7901, SNU-1 and SNU-16. Moreover, the quantitative analysis results by real-time PCR confirmed that expression of FGFR4 mRNA in the MKN45 cells was highest among the 4 common GC cell lines (Fig. 1B). In addition, expression of FGFR4 protein in the MKN45 and SGC7901 cells was obviously higher than that in the other 2 cell lines; in particular, high expression of FGFR4 protein was noted in the MKN45 cells (Fig. 1E). Therefore, the MKN45 cell line was chosen to undergo subsequent assays.

PD173074, 5-Fu and FGF19 have an impact on the cell viability of MKN45 cells. To evaluate the growth effect of the different agents on MKN45 cells, CCK-8 was used to detect the cell viability using a microplate reader. As the concentration of $\mathrm{PD}$ increased, the cell viability progressively decreased; the $\mathrm{IC}_{50}$ value was $9.856 \pm 1.046 \mu \mathrm{M}$ (Fig. 2A). A similar trend was found when MKN45 cells were treated with different concentrations of 5-Fu; the $\mathrm{IC}_{50}$ was $395.45 \pm 5.46 \mu \mathrm{M}$ (Fig. 2B). As shown in Fig. 2C, the inhibitory effect on the
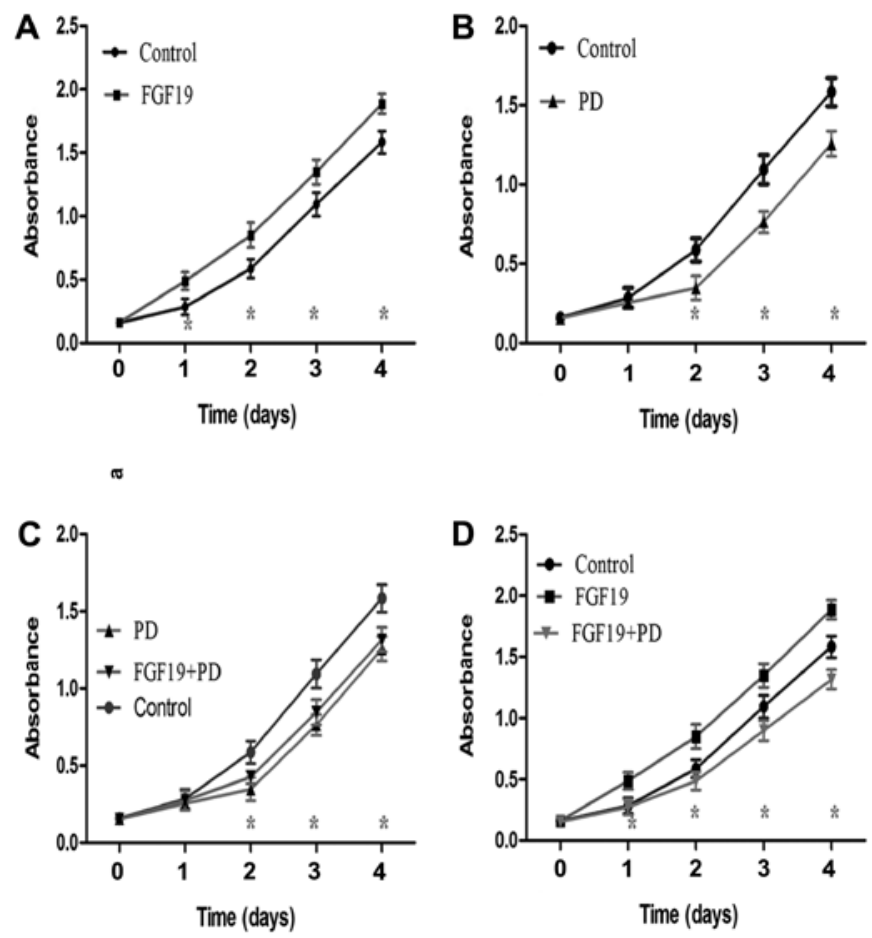

Figure 3. Influence of single agent treatment and combination of PD173074 and FGF19 on the proliferation of MKN45 cells as detected by CCK-8. Cell absorbance was consecutively measured for 5 days. (A) FGF19 markedly increased the proliferative ability of MKN45 cells (Student's t-test; $\left.{ }^{*} \mathrm{P}<0.05\right)$ while (B) PD obviously weakened it when compared with the control group (Student's t-test, ${ }^{*} \mathrm{P}<0.05$ ). (C) The proliferation of cells treated with PD for $2 \mathrm{~h}$ prior to adding FGF19, was similar with that of the single PD group (Student's t-test; $\mathrm{P}>0.05$ ) while the proliferation of the two groups was significantly lower than that of the control group (oneway ANOVA; $\left.{ }^{*} \mathrm{P}<0.05\right)$. (D) The proliferation of the single agent FGF19 treatment group was markedly increased when compared with that of the control group as well as that of the cells treated with FGF19 for $2 \mathrm{~h}$ prior to adding PD (one-way ANOVA; ${ }^{*} \mathrm{P}<0.05$ ). All experiments were performed in triplicate and were repeated at least three times.

cell viability of MKN45 cells was more significant following the combination treatment of 5-Fu and PD than that following single administrations at different concentrations; the differences achieved statistical significance (one-way ANOVA and Dunnett's test; $\mathrm{P}<0.05)$. Moreover, as the concentration of FGF19 increased, the cell viability gradually rose and the approximate linear relation was shown at concentrations of 12.5-100 ng/ml (Fig. 2D). In order to carry out subsequent assays, the appropriate concentration of each agent was chosen in the linear area. According to the above results, the final suitable concentrations of PD, 5-Fu and FGF19 were $10 \mu \mathrm{M}$, $400 \mu \mathrm{M}$ and $50 \mathrm{ng} / \mathrm{ml}$, respectively.

Single agent treatments and the combination of PD173074 and FGF19 influence the proliferation of MKN45 cells. To verify the effect of PD on the proliferation of gastric cancer cells mainly by inhibiting the FGFR4 pathway, FGF19, a special agonist of FGFR4, was introduced to clarify this issue. The 4 treatment groups were categorized as follows: group 1 (FGF19, $50 \mathrm{ng} / \mathrm{ml}$ ); group 2 (PD, $10 \mu \mathrm{M}$ ); group 3 (cells were treated with PD for $2 \mathrm{~h}$ prior to adding FGF19); group 4 (cells were treated with FGF19 for $2 \mathrm{~h}$ prior to adding PD). MKN45 cells 


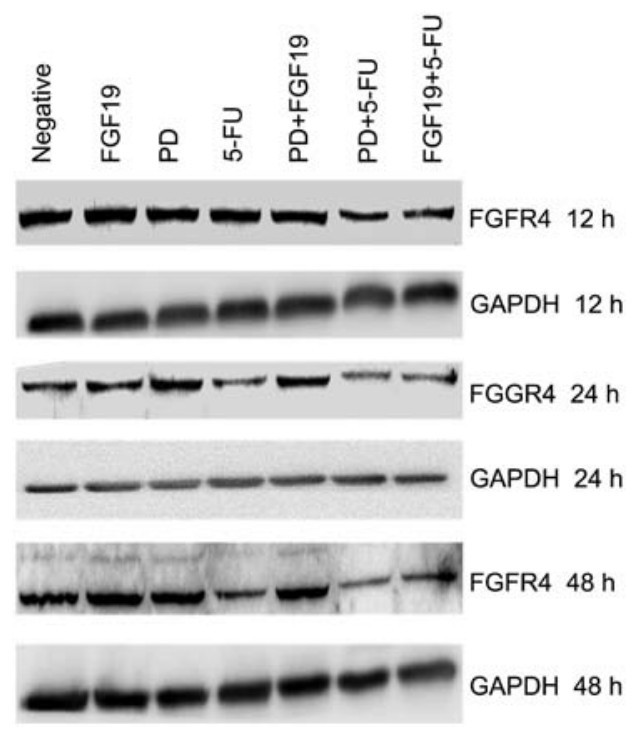

Figure 4. Single agent treatment and combinations of FGF19, PD173074 and 5-Fu impact the expression of FGFR4 in MKN45 cells. Western blot analysis was used to detect FGFR4 in the different treatment groups at 12, 24 and $48 \mathrm{~h}$. FGFR4 expression was reduced following single agent treatment with 5-Fu and following combination treatments of 5-Fu and FGF19/PD. GAPDH served as the loading control. All data shown are representative of at least three independent experiments.

without any treatment served as the control group. CCK-8 was used to detect cell absorbance to evaluate cell proliferation.

FGF19 markedly increased the proliferative ability of the MKN45 cells when compared with that of the control group (Fig. 3A, Student's t-test; $\mathrm{P}<0.05$ ), particularly from day 2 to day 5 after CCK-8 detection. Compare to the control, PD obviously weakened the proliferative ability of the MKN45 cells from day 3 to day 5 after CCK-8 assay (Fig. 3B, Student's t-test; $\mathrm{P}<0.05$ ). As shown in Fig. $3 \mathrm{C}$, the effect of treatment with PD for $2 \mathrm{~h}$ prior to adding FGF19 (group 3) on the proliferation of MKN45 cells was similar to that of cells treated with $10 \mu \mathrm{M}$ PD (group 2) (Student's t-test; P>0.05), although a significant difference was noted when the two groups were compared with control from day 3 to day 5 after CCK- 8 detection (Fig. 3C, one-way ANOVA; $\mathrm{P}<0.05$ ). However, the proliferation of MKN45 cells was more significantly increased following single agent FGF19 treatment than that following treatment of FGF19 for $2 \mathrm{~h}$ prior to adding PD (group 4) and the control group from day 2 to day 5 after CCK-8 assay (Fig. 3D, one-way ANOVA; P<0.05).

Single agent treatment and combinations of FGF19, PD173074 and 5-Fu affect the expression of FGFR4 in MKN45 cells. To clarify whether the different reagents affect the expression of FGFR4 in GC cells, western blot analysis was applied to detect the expression of FGFR4 in MKN45 cells following treatment with the single agents and combinations of FGF19, PD and 5-Fu for 12, 24 and $48 \mathrm{~h}$, respectively. As shown in Fig. 4, the single agent treatments and the combination of FGF19 and PD had no obvious influence on the expression of FGFR4 in the MKN45 cells when compared with the negative control. However, FGFR4 expression was markedly weakened following single agent treatment with 5-Fu, the combination of
Table I. Effect on the apoptosis rate following different treatments in the MKN45 cells for $24 \mathrm{~h}$ (one-way ANOVA).

\begin{tabular}{lrr}
\hline Treatment & $\begin{array}{r}\text { Apoptosis } \\
\text { rate }(\%)^{\mathrm{a}}\end{array}$ & P-value $^{\mathrm{b}}$ \\
\hline Control & $12.70 \pm 1.06$ & \\
FGF19 $(50 \mathrm{ng} / \mathrm{ml})$ & $9.25 \pm 0.89$ & 0.069 \\
PD $(10 \mu \mathrm{M})$ & $31.23 \pm 2.09^{\mathrm{c}}$ & 0.000 \\
5 -FU $(400 \mu \mathrm{M})$ & $42.64 \pm 1.86^{\mathrm{c}}$ & 0.000 \\
PD $(10 \mu \mathrm{M})+\mathrm{FGF} 19(50 \mathrm{ng} / \mathrm{ml})$ & $25.44 \pm 1.86^{\mathrm{c}}$ & 0.000 \\
PD $(10 \mu \mathrm{M})+5-\mathrm{Fu}(400 \mu \mathrm{M})$ & $52.97 \pm 1.97^{\mathrm{c}}$ & 0.000 \\
FGF19 $(50 \mathrm{ng} / \mathrm{ml})+5-\mathrm{Fu}(400 \mu \mathrm{M})$ & $41.35 \pm 1.35^{\mathrm{c}}$ & 0.000 \\
\hline
\end{tabular}

One-way ANOVA: ${ }^{a} \mathrm{~F}$-value, 327.82; $\mathrm{P}=0.000$. ${ }^{\mathrm{b}}$ Dunnett's test was used for comparison among groups. 'Significant difference was achieved in the different treatment groups when compared with the control group $(\mathrm{P}<0.05)$. Three individual experiments were performed. Results are expressed as means \pm standard deviation.

5-Fu and PD as well as the combination of 5-Fu and FGF19, in particular following treatment for 24 and $48 \mathrm{~h}$ (Fig. 4). In other words, 5-Fu reduced the expression of FGFR4 in MKN45 cells.

Single agent treatment and combinations of FGF19, PD173074 and 5-Fu impact the apoptosis rate of MKN45 cells. To explore whether the different reagents impact the apoptosis of GC cells, Annexin V and PI staining and flow cytometry were used to detect the apoptosis rate of MKN45 cells following treatments with the single agents and combinations of FGF19, PD and 5-Fu for $24 \mathrm{~h}$. The apoptosis rate of the control group was $12.70 \pm 1.06 \%$. An increased apoptosis rate was noted in all treatment groups expect for cells treated with the single agent FGF19, and the differences were significantly (Table I; one-way ANOVA, F-value $=327.82$; $\mathrm{P}<0.000$; Dunnett's test, $\mathrm{P}<0.05$ ). In comparison of the rates, the apoptosis rate following the combination of 5-Fu and PD, the highest following all treatments, was obviously increased when compared with the apoptosis rate following treatment with the single agent treatments of 5-Fu and PD (Fig. 5).

Different treatments affect the expression of signaling pathway and downstream effector molecules in MKN45 cells. To explore how different treatments affect the biological behavior of MKN45 cells, expression of cell signaling pathway-associated molecules (Akt, p-Akt and p-ERK) and apoptosis-associated molecules (caspase- 3 and Bcl-xl) was determined to clarify the mechanism.

As shown in Fig. 6, expression of p-Akt was increased in the single agent PD and single FGF19 treatment groups while no obviously change was noted in the 5-Fu single agent treatment group when compared with the negative control. However, the combination of 5-Fu and PD decreased p-Akt expression. As for Akt, there were no marked changes in the different treatment groups. Furthermore, compared to the negative control, p-ERK expression was dramatically decreased in the single agent and combination treatment groups of 5-Fu and PD. 

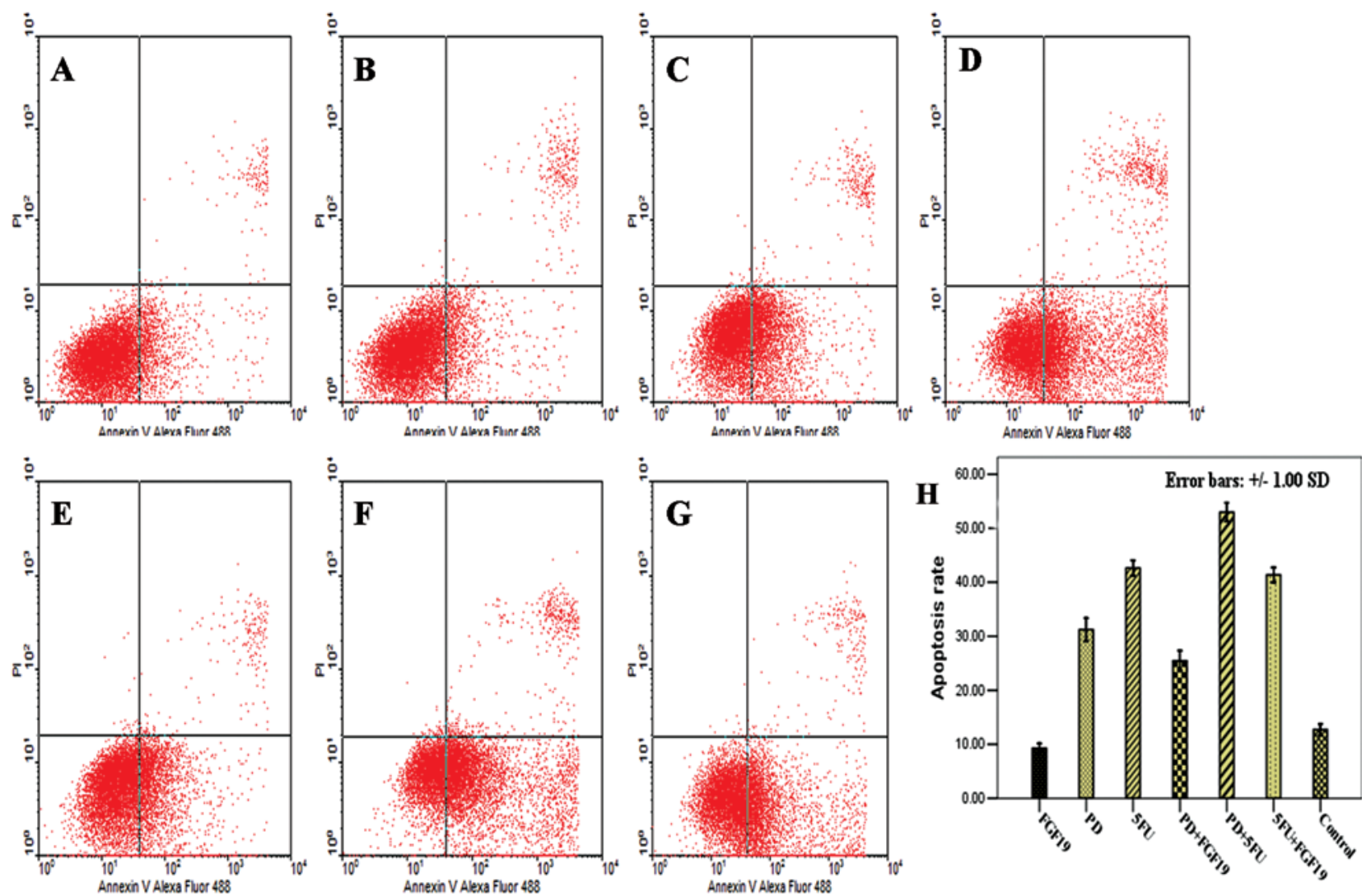

Figure 5. Flow cytometry was applied to evaluate the effect of the single and combination treatments of FGF19, PD173074 and 5-Fu on the apoptosis rate of MKN45 cells. Apoptosis was assessed in the: (A) negative control; (B) following single agent FGF19 treatment; (C) following single agent PD treatment; (D) following single agent 5-Fu treatment; (E) following treatment with the combination of PD and FGF19; (F) following treatment with the combination of PD and 5-Fu; and $(\mathrm{G})$ following treatment with the combination of 5-Fu and FGF19. $(\mathrm{H})$ Apoptosis rate following the different treatments. All data shown are representative of at least three independent experiments.

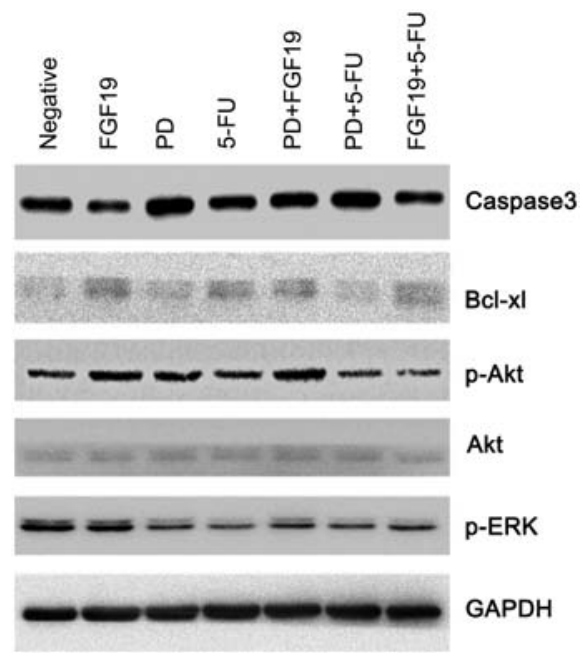

Figure 6. Western blot analysis revealed the impact of different treatments for $24 \mathrm{~h}$ on the expression of signaling pathway- (Akt, p-Akt and p-ERK) and apoptosis-associated molecules (caspase-3 and Bcl-xl) in MKN45 cells. GAPDH served as the loading control. Seven treatment groups included the negative control, single FGF19 group, single PD group, single 5-Fu group combination group of PD and FGF19, combination group of PD and 5-Fu and combination group of 5-Fu and FGF19. Obvious change in expression following the different treatments was noted. All data shown are representative of at least three independent experiments.

When compared with the negative control, expression of caspase- 3 was obviously increased in the single agent PD group while expression of Bcl-xl was prominently weakened. Furthermore, compared to treatment with the single agent 5-Fu, expression of caspase- 3 was markedly strengthened while expression of Bcl-xl was notably reduced following the combination treatment of 5-Fu and PD. In other words, the combination of 5-Fu and PD had a synergistic effect on promoting apoptosis. As for the FGF19 group, expression of effector molecules was contrary to that noted in the single agent PD treatment group. However, in the combination group of FGF19 and PD, expression of effector molecules was similar to that in the single agent PD treatment group when compared with that in the single FGF19 treatment group. Therefore, PD influenced the effect of FGF19 by inhibiting FGFR4.

\section{Discussion}

Recently, molecular-targeted drugs are prevalently investigated in gastrointestinal tumors. However, no other targeted drug has achieved a breakthrough in gastric cancer apart from Herceptin. Therefore, it is urgently necessary that novel molecular markers and therapeutic targets should be explored and investigated. In addition to EGFR, VEGF and Her-2, the FGFR family has currently received increased attention for the treatment of malignant tumors, attracting the interest of numerous investigators. Among them, FGFR4, a member of the FGFR family, has become a popular research molecule in various tumors. However, the effect of FGFR4 on GC has seldom been 
studied, and research is urgently required to fully clarify its role. Our previous research revealed that FGFR4 leads to GC progression by influencing proliferation and causing an antiapoptotic effect, suggesting FGFR4 as a new drug target for the treatment of GC (6). Based on the clinical application of Herceptin, the aim of the present study was to initially explore whether single agent treatments and a combination of 5-Fu and PD173074 impact the biological behavior of GC cells as well as the mechanism of the two-drug combination using a series of functional research methods in vitro.

PD was found to inhibit wild-type and constitutively activated mutant FGFR3 autophosphorylation in multiple myeloma, which was associated with decreased viability and tumor cell growth arrest (8). Selective FGFR inhibitor PD blocks H-510 and H-69 SCLC proliferation and clonogenic growth in a dose-dependent fashion; PD was also found to significantly strengthen the effect of cisplatin and increase apoptosis (9). PD weakens the activity of FGFR4 and reduces the phosphorylation FGFR4 in breast cancer and medullary thyroid cancer $(10,11)$. In the thyroid cancer cell lines MRO and ARO with high FGFR4 expression, cell proliferation gradually decreased with increasing PD concentration. SCID mice implanted with aggressively growing MRO cells that endogenously express significant amounts of FGFR-4 demonstrated a significant decrease in tumor size following PD treatment, suggesting that PD inhibits the activity of FGFR4 in thyroid carcinoma (12). Specific and complete reversal of FGF19stimulated AFP production by PD justified that inhibiting the activity of FGFR4 may be the most important mechanism of PD in hepatocellular carcinoma; PD promoted apoptosis and increased chemotherapy sensitivity in HCC (7).

Among more than 20 FGF ligands, only FGF19 can directly and specifically combine to FGFR4 $(13,14)$. Moreover, no matter whether $\beta$-Klotho was present, FGF19 led to biological effects by combining with FGFR4 (15). FGF19-induced hepatocyte proliferation was mediated through FGFR4 activation (16). FGF19 reduced the expression of cyp7A1 mRNA in liver tissue by FGFR4 activation (17). Furthermore, 5-Fu is a cell-cycle specific chemotherapy drug and mainly arrests cells in the $\mathrm{S}$ phase.

As shown in Fig. 1, expression of FGFR4 in MKN45 cells, at the mRNA and protein levels, was highest among the 4 common gastric cancer cell lines. Thus, MKN45 cells were chosen for the subsequent assays. As the concentration of 5-Fu and PD increased, following administration as single agents, the cell viability of MKN45 cells in both groups gradually declined. Moreover, the growth inhibition of MKN45 cells following the combination treatment of 5-Fu and PD was more significant than that in the single agent treatments, suggesting that the combination of 5-Fu and PD had synergistic effects on the growth inhibition of GC cells.

In the present study, the effects of the single agent treatment and the combination of PD and FGF19 on the proliferation of MKN45 was detected by CCK-8. The cells treated with PD for $2 \mathrm{~h}$ prior to adding FGF19, PD could almost inhibit cell proliferation as that promoted by FGF19 (Fig. 3C). However, when cells were treated with FGF19 for $2 \mathrm{~h}$ prior to adding PD, the proliferation rate of the combination group was significantly less than that of the single agent FGF19 treatment group. Therefore, in MKN45 cells with high expression of FGFR4,
PD decreased the proliferation of GC cells mainly through inhibiting the activity of FGFR4, which is in accordance with the research in thyroid cancer and hepatocellular carcinoma $(7,12)$.

A significant findings was that FGFR4 expression was obviously weakened in the single agent 5-Fu treatment group and in the combination 5-Fu and PD/FGF19 treatment groups, particularly after treatments for 24 and $48 \mathrm{~h}$ (Fig. 4). This indicated that $5-\mathrm{Fu}$ reduced FGFR4 expression in the GC cells, beyond the effect of FGF19 and PD. This finding clarifies the mechanism of the synergistic effect in the combination group of 5-Fu and PD, through decreasing FGFR4 expression and inhibiting the activity of FGFR4, respectively.

Compared with the negative control group, single agent treatments of PD and 5-Fu increased the apoptosis rate of MKN45 cells. Furthermore, the apoptosis rate following treatment with the combination of 5-Fu and PD was much higher than that in the single administration groups, suggesting that PD strengthens the activity of 5-Fu in regulating apoptosis (Fig. 5). Western blot analysis showed that PD increased caspase-3 expression and reduced Bcl-xl expression when compared with the negative control, implying that PD promoted MKN45 cell apoptosis. Roidl et al (18) reported that an FGFR4 inhibitor reduced Bcl-xl expression in breast cancer cell lines, dramatically increased the apoptosis rate of breast cancer cells and strengthened the sensitivity to doxorubicin, which was in line with our results. The apoptosis rate in the combination group of PD and FGF19, similar to the single agent PD treatment group, was between the values for the single agent treatment groups of the two drugs. This indicates that PD may increase the apoptosis of GC by inhibiting the FGF19/FGFR4 pathway. Furthermore, Drafahl et al (19) found that activation of FGFR4 may lead to a negative effect on the $N F-\kappa B$ pathway, suggesting that $N F-\kappa B$ may be one of the signal pathways influencing apoptosis.

Our research also investigated the effects of different treatments on the expression of signaling pathway molecules in MKN45 cells. Except for FGF19, p-ERK expression was decreased with different levels in all other groups compared to the negative control (Fig. 6). Roidl et al (18) showed that FGFR4 inhibitor reduced p-ERK expression in breast cancer cells and inhibited p-ERK activated by FGF19, which was in accordance with our results. Akt expression had no obvious difference. However, p-Akt expression was markedly increased following the single agent treatments and the combination of FGF19 and PD while p-Akt expression was slightly decreased following treatment with the combinations of 5-Fu and FGF19/ $\mathrm{PD}$ when compared to the negative group, implying that $\mathrm{p}-\mathrm{Akt}$ may have an effect on the FGF19/FGFR4 signaling pathway, which needs to be further clarified.

In conclusion, the present study initially explored the effect and the mechanism of single agent treatments and combinations of PD173074, FGF19 and 5-Fu on the biological characteristics of gastric cancer cells. Inhibiting the activity of FGFR4 may be one of the vital important mechanisms by which PD inhibits GC cell proliferation and increases the rate of apoptosis. 5-Fu reduces the expression of the FGFR4 protein, which may provide a basis for the treatment of gastric cancer by a combination of 5-Fu and PD. Furthermore, the combination of 5-Fu and PD had a synergistic effect in reducing proliferation 
and increasing the apoptosis rate in gastric cancer cells. The results of the present study may contribute to the development of the new target drug PD173074 in combination with 5-Fu, similar to Heceptin, for the clinical treatment of gastric cancer. Obviously, our results should be verified by further research in vitro and in vivo.

\section{Acknowledgements}

The present study was supported by the Department of Gastrointestinal Surgery, The First Affiliated Hospital, Zhengzhou University and the National Natural Science Foundation of China (grant no. 81201955).

\section{References}

1. Kamangar F, Dores GM and Anderson WF: Patterns of cancer incidence, mortality, and prevalence across five continents: defining priorities to reduce cancer disparities in different geographic regions of the world. J Clin Oncol 24: 2137-2150, 2006.

2. Katoh M: Genetic alterations of FGF receptors: an emerging field in clinical cancer diagnostics and therapeutics. Expert Rev Anticancer Ther 10: 1375-1379, 2010.

3. Wang J, Stockton DW and Ittmann M: The fibroblast growth factor receptor-4 Arg388 allele is associated with prostate cancer initiation and progression. Clin Cancer Res 10: 6169-6178, 2004.

4. Eswarakumar VP, Lax I and Schlessinger J: Cellular signaling by fibroblast growth factor receptors. Cytokine Growth Factor Rev 16: 139-149, 2005.

5. Ye Y, Shi Y,Zhou Y, et al: The fibroblast growth factor receptor-4 Arg388 allele is associated with gastric cancer progression. Ann Surg Oncol 12: 3354-3361, 2010.

6. Ye YW,Zhou Y, Yuan L, et al: Fibroblast growth factor receptor 4 regulates proliferation and antiapoptosis during gastric cancer progression. Cancer 117: 5304-5313, 2011.
7. Ho HK, Pok S, Streit S, et al: Fibroblast growth factor receptor 4 regulates proliferation, anti-apoptosis and alpha-fetoprotein secretion during hepatocellular carcinoma progression and represents a potential target for therapeutic intervention. J Hepatol 50: 118-127, 2009.

8. Trudel S, Ely S, Farooqi Y, et al: Inhibition of fibroblast growth factor receptor 3 induces differentiation and apoptosis in $\mathrm{t}(4 ; 14)$ myeloma. Blood 103: 3521-3528, 2004.

9. Pardo OE, Latigo J, Jeffery RE, et al: The fibroblast growth factor receptor inhibitor PD173074 blocks small cell lung cancer growth in vitro and in vivo. Cancer Res 69: 8645-8651, 2009.

10. Koziczak M and Hynes NE: Cooperation between fibroblast growth factor receptor- 4 and ErbB2 in regulation of cyclin D1 translation. J Biol Chem 279: 50004-50011, 2004.

11. Ezzat S, Huang P, Dackiw A, et al: Dual inhibition of RET and FGFR4 restrains medullary thyroid cancer cell growth. Clin Cancer Res 11: 1336-1341, 2005.

12. St. Bernard R, Zheng L, Liu W, et al: Fibroblast growth factor receptors as molecular targets in thyroid carcinoma. Endocrinology 146: 1145-1153, 2005.

13. Xie MH, Holcomb I, Deuel B, et al: FGF-19, a novel fibroblast growth factor with unique specificity for FGFR4. Cytokine 11: 729-735, 1999.

14. Harmer NJ, Pellegrini L, Chirgadze D, et al: The crystal structure of fibroblast growth factor (FGF) 19 reveals novel features of the FGF family and offers a structural basis for its unusual receptor affinity. Biochemistry 43: 629-640, 2004.

15. Wu X, Ge H, Lemon B, et al: Selective activation of FGFR4 by an FGF19 variant does not improve glucose metabolism in ob/ob mice. Proc Natl Acad Sci USA 106: 14379-14384, 2009.

16. Wu X, Ge H, Lemon B, et al: FGF19-induced hepatocyte proliferation is mediated through FGFR4 activation. J Biol Chem 285: 5165-5170, 2010 .

17. Inagaki T, Choi M, Moschetta A, et al: Fibroblast growth factor 15 functions as an enterohepatic signal to regulate bile acid homeostasis. Cell Metab 2: 217-225, 2005.

18. Roidl A, Berger HJ, Kumar S, et al: Resistance to chemotherapy is associated with fibroblast growth factor receptor 4 up-regulation. Clin Cancer Res 15: 2058-2066, 2009.

19. Drafahl KA, McAndrew CW, Meyer AN, et al: The receptor tyrosine kinase FGFR4 negatively regulates NF-kappaB signaling. PLoS One 5: e14412, 2010. 